\title{
Desarrollo de la competencia de razonamiento y argumentación en estudiantes de quinto grado de Educación Básica Primaria
}

\section{Development of competition of reasoning and argumentation in fifth grade of elementary Basic Education Students}

\author{
Eusebio Campo Peña, \\ Magister en Educación Universidad Autónoma del Caribe, Especialista en Pedagogía de las Ciencias de la Universidad Simón Bolívar de Barranquilla, \\ Especialista en Estudios Pedagógicos de la CUC, Licenciado en Matemáticas y Física de la Universidad del Atlántico, Tutor del programa del MEN “Todos \\ a Aprender", Docente del Colegio Comunitario Distrital Pablo Neruda de Barranquilla, eusebio319@hotmail.com \\ Claudia Devia Miranda, \\ Magister en Educación Universidad Autónoma del Caribe, Especialista en Estudios Pedagógicos de la CUC, Psicóloga de la Universidad Metropolitana \\ de Barranquilla, Coordinadora de la IETT Simón Bolívar de Puerto Colombia, Atlántico, claudiadevia2003@yahoo.com.mx
}

\begin{abstract}
RESUMEN
Se presenta la problemática centrada en la dificultad que presentan los estudiantes en su desempeño respecto a la competencia matemática de razonamiento y argumentación; esto evidenciado en los bajos resultados en pruebas externas (SABER y prueba diagnóstica Programa Todos a Aprender) así como en un diagnóstico aplicado a una muestra de cuatros grupos, que oscilaba en 38 estudiantes de $5^{\circ}$ del IETT Simón Bolívar de Puerto Colombia. Se aborda como objetivo diagnosticar la problemática a partir de identificar las falencias relacionadas con la competencia de razonamiento y argumentación en la resolución de problemas. Por tanto se realiza una investigación cuantitativa orientada bajo un diseño pre-experimental de grupo intacto con pre-test y pos-test, que tiene como sustentos teóricos los aportes de Carlos Vasco en cuanto al enfoque de competencias y G. Polya en lo referente a la resolución de problemas. A partir del diagnóstico realizado a docentes en cuanto a los referentes que abordan para la enseñanza,y a la revisión de los resultados de evaluaciones locales, nacionales e internacionales aplicadas a los estudiantes, se denotan falencias en lo referente al desarrollo de la competencia matemáticas de razonamiento y argumentación; hecho que resalta la necesidad de elaborar e implementar estrategias pedagógicas que propendan por el desarrollo de las competencias matemáticas y contribuyan a fortalecer el trabajo de los docentes en aras de potenciar en los estudiantes dichas competencias.
\end{abstract}

Palabras Clave: Competencias matemáticas, razonamiento y argumentación, estrategia pedagógica, resolución de problemas.

\begin{abstract}
Arises issues focused on the difficulty that students present their performance with respect to the mathematical competition of reasoning and argumentation; This evidenced in the low performance in external tests (learn and test diagnostic program all to learn) as well as in a diagnosis applied to a sample of four groups, ranging in 38 students of 5th of the IETT Simon Bolivar de Puerto Colombia. Deals with objective diagnose the problem from identifying the shortcomings related to the competence of reasoning and argumentation in the resolution of problems. Therefore is a quantitative research under a pre-experimental design by intact group with pre-test and pos-test, whose livelihoods theoretical contributions from Carlos Vasco in terms of competences and G. Polya approach in relation to problem solving. From the diagnosis carried out to teachers regarding the references that dealt with teaching, and the revision of the results of local, national and international assessments applied to students, is denote shortcomings in relation to the development of mathematics competence of reasoning and argumentation; fact that highlights the need to develop and implement teaching strategies that foster the development of mathematical skills and contribute to strengthen the work of teachers in order to enhance these skills in students.
\end{abstract}

Key Words: Math skills, reasoning and argumentation, pedagogical strategy, troubleshooting. 


\section{Introducción}

La educación como proceso humano complejo se ha ido transformando a lo largo de la historia, apoyado en distintos enfoques y paradigmas que han permeado las metodologías y modelos a partir de los cuales se ha pretendido la formación del hombre. Hoy día, es de suma relevancia el enfoque educativo de formación por competencias; por ende, es pertinente hacer una revisión de dicho concepto y su evolución con el fin de sentar las bases conceptuales del presente trabajo investigativo.

El concepto competencia como tal se comenzó a estructurar en la década del sesenta con base en dos aportaciones: la lingüística de Chomsky y la psicología conductual de Skinner. Desde lo conductual ha tenido notables desarrollos en el campo de la gestión del talento humano en las organizaciones y a mediados de la década de los años noventa se ha implementado en instituciones educativas de varios países, buscando con ello formar personas con ciertas competencias que les posibiliten un mayor impacto en la inserción laboral.

Aportes de Vigotsky (1985), denotando las competencias como "acciones situadas que se definen en relación con determinados instrumentos mediadores" (Hernández et al., 1998, p. 14), resaltan su carácter contextual. De igual forma, desde la psicología cognitiva se destaca la teoría de las inteligencias múltiples de Gardner $(1987,1997)$ la cual da un apoyo teórico sustancial a la comprensión de las competencias en su dimensión cognoscitiva (Tobón, 2005). Se tienen también las contribuciones de Sternberg (1997) en torno a la inteligencia práctica, la cual se refiere a la capacidad que han de tener las personas para desenvolverse con inteligencia en las situaciones de la vida.

Como sustento teórico - conceptual en el trabajo investigativo se toman como referente las concepciones siguientes:

La competencia se define como conjunto de conocimientos, habilidades, actitudes, comprensiones y disposiciones cognitivas, socio-afectivas y psicomotoras apropiadamente relacionadas entre sí para facilitar el desempeño flexible, eficaz y con sentido de una actividad en contextos relativamente nuevos y retadores (Ministerio De Educación Nacional, MEN, 2006).

El concepto anterior, al ser el soporte del enfoque de competencias en el contexto educativo nacional, es fundamental dentro del proceso investigativo desarrollado en el presente trabajo, puesto que se vincula con otros referentes nacionales que orientan el quehacer docente como lo son los lineamientos curriculares y los estándares básicos de competencias. Además, denota la complejidad de la competencia no limitándola únicamente a lo cognitivo, sino resaltando también aspectos socio - afectivos y psicomotores, asociado a desempeño flexible y significativo en distintos contextos.
Para Tobón (2006) las competencias son procesos complejos de desempeño con idoneidad en un determinado contexto, con responsabilidad. Dicho concepto es pertinente, porque además de reafirmar lo expuesto por el Ministerio de Educación Nacional y sintetizarlo de manera acertada, lo complementa puntualizando dos aspectos fundamentales: idoneidad y responsabilidad. El primero relacionado con el desempeño en la resolución de problemas con criterios de eficacia, eficiencia, efectividad, pertinencia y apropiación. El segundo como el ejercicio ético de prever las consecuencias del desempeño.

Ahora bien, la problemática que suscita el presente trabajo investigativo se centra en los bajos resultados académicos de los estudiantes de básica primaria en al área de matemáticas denotado por bajos niveles de desarrollo de competencias a su vez evidenciados en pruebas estandarizadas nacionales (SABER y prueba diagnóstica del programa PTA: "Todos a aprender"Programa de transformación de la calidad. MEN).

Dando una mirada global a la problemática, denótese como se encuentra Colombia con relación al desarrollo de las competencias matemáticas de sus estudiantes frente a otros países. Para tal cuestión, el referente de análisis serán las pruebas internacionales PISA que Colombia aplicó en el 2009, y donde se definen las competencias matemáticas de la siguiente forma: "La capacidad de formular, emplear e interpretar la matemática en diversos contextos. Incluye el razonamiento matemático y el uso de conceptos, procedimientos, hechos y herramientas para describir, explicar y predecir fenómenos. Esta competencia permite al estudiante reconocer el rol que juega la matemática en el mundo, tomar decisiones y emitir juicios bien fundamentados como ciudadano constructivo".

En cuanto a los resultados de Colombia en la prueba PISA 2009, se denota la Tabla 1.

Lo anterior muestra entonces (ver tabla 1) a Colombia en una posición 58 entre 65 países evaluados, lo que refrenda entonces los bajos resultados de los estudiantes en lo concerniente a las competencias matemáticas.

Por su parte, como referente nacional, las pruebas saber muestran también un panorama similar evidenciado en el diagnóstico nacional de calidad de la educación presentado como sustento del Programa Nacional de Transformación de la Calidad "Todos a Aprender". Antes es pertinente reconocer como los referentes nacionales denotan las competencias matemáticas y los niveles de desempeño.

A continuación, se presentan tabla2. y las figuras de gráficos 1, 2 y 3 , para contextualizar teóricamente los resultados en esos referentes nacionales: 
Tabla 1. Puntajes promedio en matemáticas pruebas PISA 2009.

\begin{tabular}{|c|c|c|c|c|c|}
\hline Pais & Promedio & Desviación estándar & Pais & Promedio & Desviación estándar \\
\hline Shangháai & 600 & 103 & \multirow{33}{*}{$\begin{array}{l}\text { Portugal } \\
\text { Espanaa } \\
\text { Italia } \\
\text { Letonia } \\
\text { Lituania } \\
\text { Rusia } \\
\text { Grecia } \\
\text { Croacia } \\
\text { Dubái } \\
\text { Israel } \\
\text { Turquía } \\
\text { Serbia } \\
\text { Azerbaiyán } \\
\text { Bulgaria } \\
\text { Rumania } \\
\text { Uruguay } \\
\text { Chile } \\
\text { Taillandia } \\
\text { México } \\
\text { Trinidad y Tobago } \\
\text { Kazajistán } \\
\text { Montenegro } \\
\text { Argentina } \\
\text { Jordania } \\
\text { Brasil } \\
\text { Colombia } \\
\text { Albania } \\
\text { Túnez } \\
\text { Indonesia } \\
\text { Qatar } \\
\text { Perú } \\
\text { Panamá } \\
\text { Kirguistán } \\
\end{array}$} & 487 & 91 \\
\hline Singapur & 562 & 104 & & 483 & 91 \\
\hline Hong Kong & 555 & 95 & & 483 & 93 \\
\hline Corea & 546 & 89 & & 482 & 79 \\
\hline Taipei & 543 & 105 & & 477 & 88 \\
\hline Finlandia & 541 & 82 & & 468 & 85 \\
\hline Liechtenstein & 536 & 88 & & 466 & 89 \\
\hline Suiza & 534 & 99 & & 460 & 88 \\
\hline Japón & 529 & 94 & & 453 & 99 \\
\hline Canadá & 527 & 88 & & 447 & 104 \\
\hline Paises Bajos & 526 & 89 & & 445 & 93 \\
\hline Macao & 525 & 85 & & 442 & 91 \\
\hline Nueva Zelanda & 519 & 96 & & 431 & 64 \\
\hline Bélgica & 515 & 104 & & 428 & 99 \\
\hline Australia & 514 & 94 & & 427 & 79 \\
\hline Alemania & 513 & 98 & & 427 & 91 \\
\hline Estonia & 512 & 81 & & 421 & 80 \\
\hline Islandia & 507 & 91 & & 419 & 79 \\
\hline Dinamarca & 503 & 87 & & 419 & 79 \\
\hline Eslovenia & 501 & 95 & & 414 & 99 \\
\hline Noruega & 498 & 85 & & 405 & 83 \\
\hline Francia & 497 & 101 & & 403 & 85 \\
\hline Eslovaquia & 497 & 96 & & 388 & 93 \\
\hline Austria & 496 & 96 & & 387 & 83 \\
\hline Polonia & 495 & 88 & & 386 & 81 \\
\hline Suecia & 494 & 94 & & 381 & 75 \\
\hline República Checa & 493 & 93 & & 377 & 91 \\
\hline Reino Unido & 492 & 87 & & 371 & 78 \\
\hline Hungría & 490 & 92 & & 371 & 70 \\
\hline \multirow{4}{*}{$\begin{array}{l}\text { Luxemburgo } \\
\text { Estados Unidos } \\
\text { Irlanda }\end{array}$} & 489 & 98 & & 368 & 98 \\
\hline & 487 & 91 & & 365 & 90 \\
\hline & 487 & 86 & & 360 & 81 \\
\hline & & & & 331 & 81 \\
\hline
\end{tabular}

OCDE (en prensa). Tomorrow's skills today. Student pertormance in PISA 2009. OCDE. Figura I.3.21.

Tabla 2. Competencias y componentes en matemáticas evaluados en las pruebas SABER 2012.

\begin{tabular}{|c|c|c|}
\hline Área & Competencias & Componentes \\
\hline Matemáticas & $\begin{array}{l}\text { - Razonamiento y argumentación } \\
\text { - Comunicación, representación y modelación } \\
\text { - Planteamiento y resolución de problemas }\end{array}$ & $\begin{array}{l}\text { - Numérico - variacional } \\
\text { - Geométrico-métrico } \\
\text { - Aleatorio }\end{array}$ \\
\hline
\end{tabular}

Tabla 3. Descripción genérica de los niveles de desempeño Prueba Saber

\begin{tabular}{|l|l|}
\multicolumn{1}{|c|}{ Nivel } & \multicolumn{1}{c|}{$\begin{array}{c}\text { Descripción } \\
\text { Un estudiante promedio ubicado en este nivel... }\end{array}$} \\
\hline Avanzado & Muestra un desempeño sobresaliente en las competencias esperadas para el área y grado evaluados. \\
Satisfactorio & $\begin{array}{l}\text { Muestra un desempeño adecuado en las competencias exigibles para el área y grado evaluados. Este } \\
\text { es el nivel esperado que todos o la gran mayoría de los estudiantes deberían alcanzar. }\end{array}$ \\
\hline Mínimo & Supera las preguntas de menor complejidad de la prueba para el área y grado evaluados. \\
Insuficiente & No supera las preguntas de menor complejidad de la prueba. \\
\hline
\end{tabular}

Fuente: Ministerio de Educación Nacional, Prueba Saber 
Figura 1. Diagnostico Nacional de calidad de la educación. Matemáticas 5º Pruebas Saber 2009.

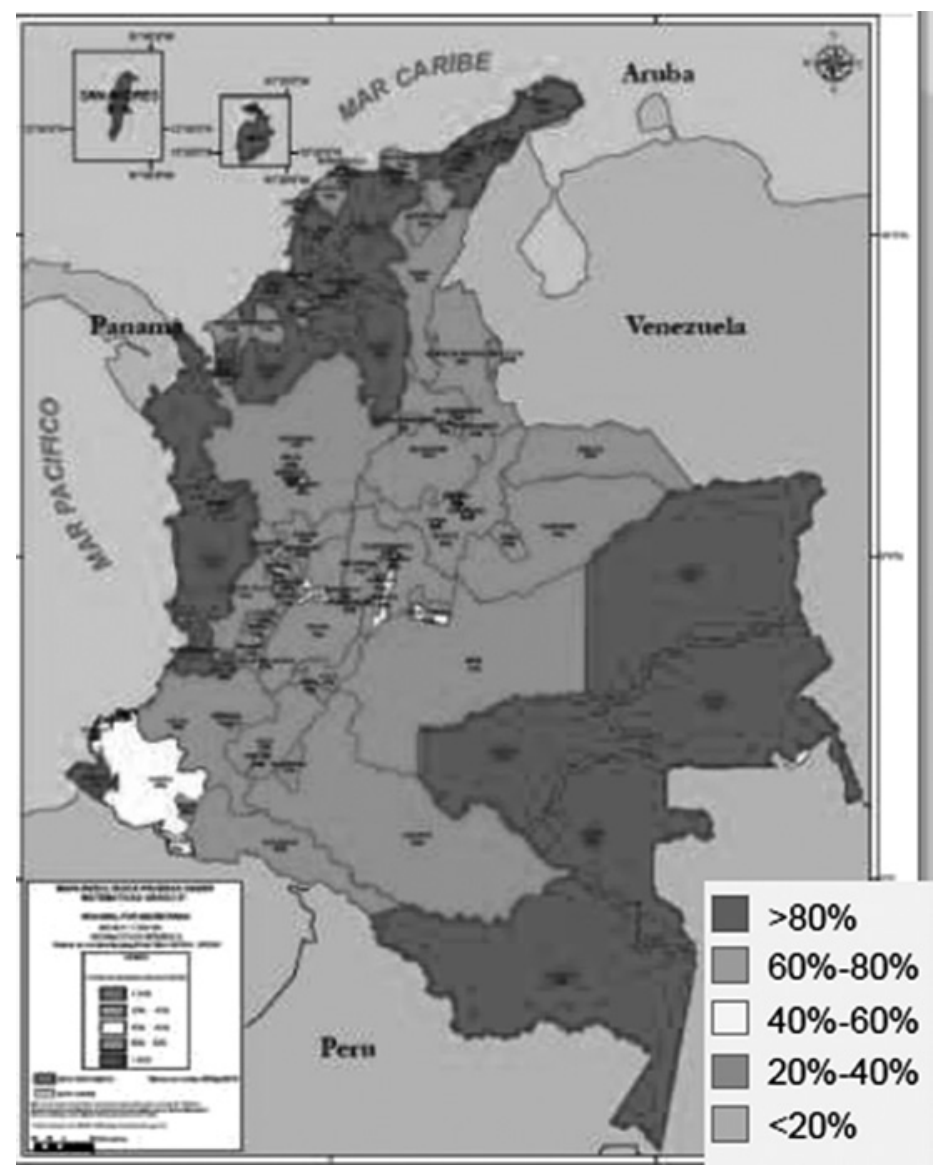

Fuente: Ministerio de Educación Nacional, 2012

Se observa entonces la situación crítica, pues la gran mayoría de departamentos del territorio nacional no se encuentran por encima de la media nacional, lo que se evidencia en los colores rojo y naranja del mapa (ver figura 1), denotando bajos niveles de desempeño de los estudiantes relacionados con las competencias matemáticas.

En el ámbito local, el referente es la Institución Educativa Técnica Turística Simón Bolívar de Puerto Colombia, Atlántico; escuela en la que se desarrolla el presente trabajo investigativo. La mirada se hace desde evaluaciones externas nacionales: prueba SABER 2009 y prueba diagnóstica 2012 del Programa de Transformación de la Calidad Educativa (Todos a Aprender) originada en el Ministerio de Educación Nacional.

En cuanto a los resultados obtenidos por los estudiantes de $5^{\circ}$ de educación básica primaria de la Institución Educativa Técnica Turística Simón Bolívar de Puerto Colombia, específicamente en lo concerniente a sus competencias matemáticas, en las pruebas anteriormente mencionadas, se muestran a continuación:
Los resultados de las pruebas Saber para $5^{\circ}$ en las Institución Educativas Técnica Turística Simón Bolívar de Puerto Colombia muestran la ubicación de su mayoría de estudiantes en los niveles de desempeño insuficiente y mínimo, con un $80 \%$. Situación refrendada con los resultados de la evaluación diagnóstica del programa Todos a Aprender con un 95,8\% de los estudiantes de $5^{\circ}$ en nivel de desempeño bajo.

Desde la investigación se buscó una aproximación a la problemática desde los docentes, aplicándoles una encuesta con el fin de reconocer sus nociones y fundamentos conceptuales y metodológicos con relación al desarrollo de competencias y la resolución de problemas, procurando a su vez direccionar la propuesta pedagógica.

Cabe resaltar que la presente investigación tubo por objetivo potenciar la competencia de razonamiento y argumentación en estudiantes de $5^{\circ}$ de educación básica primaria a través de la implementación de una propuesta pedagógica fundamentada en la resolución de problemas. Se planteo como pregunta problémica 
Figura 2. Resultados Pruebas Saber: Matemáticas $5^{\circ}$ Institución Educativa Técnica Turística Simón Bolívar de Puerto Colombia

Resultados de quinto grado en el área de matemáticas

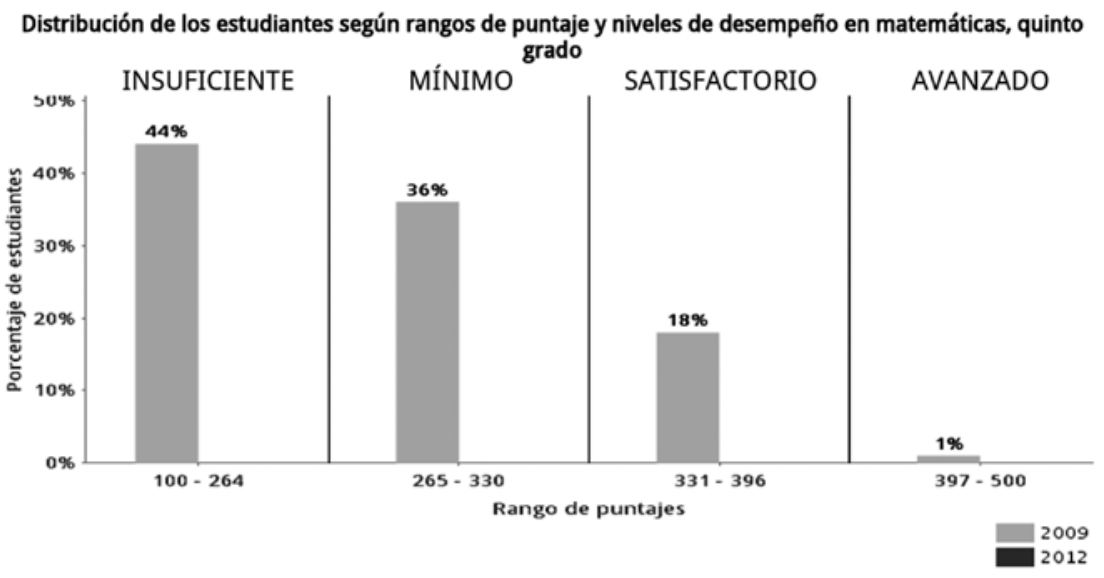

LA INFORMACIÓN DE SU INSTITUCIÓN CORRESPONDIENTE A 2012 NO ESTÁ DISPONIBLE

Fuente: MEN 2013. Prueba Saber

Figura 3. Resultados prueba diagnóstica Programa “Todos a aprender": Matemáticas $5^{\circ}$ Institución Educativa Técnica Turística Simón Bolívar de Puerto Colombia

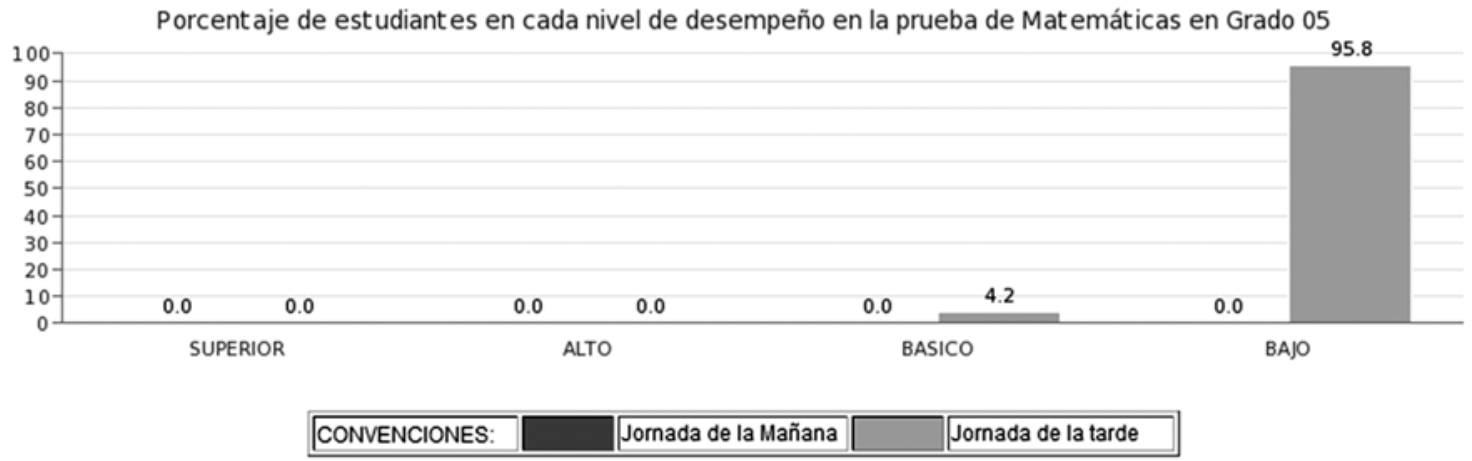

Fuente: MEN Prueba Diagnóstica 2012

la siguiente: ¿Cómo potenciar la competencia de razonamiento y argumentación en estudiantes de $5^{\circ}$ de educación básica primaria?

Teniendo como parámetro orientador la pregunta anteriormente planteada se expone la siguiente hipótesis: "Una estrategia pedagógica fundamentada en la resolución de problemas como eje dinamizador de los procesos de enseñanza y aprendizaje de las matemáticas deberá favorecer el desarrollo de las competencias de razonamiento y argumentación en estudiantes de $5^{\circ}$ de educación básica primaria”.

En cuanto a los aspectos metodológicos es relevante mencionar que la población de interés fueron los estudiantes de grado $5^{\circ}$ de Educación básica primaria de la Institución Educativa Técnica Turística Simón Bolívar de Puerto Colombia, Atlántico que cuen- ta con 4 grupos de quinto grado en la jornada vespertina, con condiciones y características equiparables para las variables del estudio, cada uno con número de estudiantes que oscilan entre 35 y 38. Se trabajó con un grupo escogido al azar para aplicar un diseño pre-experimental de grupo intacto con pre-test y pos-test, bajo los criterios de una investigación cuantitativa. Entre los instrumentos aplicados para la recolección de información se tuvo en cuenta encuestas a docentes validadas por un experto. Estas diseñadas, como ya se mencionó, con el objetivo de reconocer en los profesores sus concepciones teóricas y metodológicas en lo concerniente al desarrollo de competencias matemáticas y la resolución de problemas. De igual forma, se aplicó un taller diagnóstico a estudiantes, validado a nivel nacional pues su contenido se fundamenta en problemas originados en el Ministerio de Educación Nacional en el marco de la evaluación diagnóstica 
del Programa de Transformación de la Calidad Educativa "Todos a Aprender". El taller consta de cinco problemas que evalúan específicamente la competencia matemática de razonamiento y argumentación.

Con el ánimo de ahondar en las competencias matemáticas, la investigación se centro en el estudio y análisis del desarrollo de la competencia razonamiento y argumentación en estudiantes de $5^{\circ}$ de educación básica primaria. Por tanto, reconocer los procesos de enseñanza y aprendizaje enfocado al desarrollo de competencias matemáticas, específicamente razonamiento y argumentación, es de suma importancia, pues contribuirá al mejoramiento de los mismos y a su vez, podrá generar nuevas alternativas y perspectivas para visionar las competencias y su desarrollo desde las prácticas de aula.

\section{Metodología}

A continuación se presenta la metodología empleada en la investigación que dio origen al presente artículo: La población de interés fueron los estudiantes de grado $5^{\circ}$ de Educación básica primaria de la Institución Educativa Técnica Turística Simón Bolívar de Puerto Colombia, Atlántico que cuenta con 4 grupos de quinto grado en la jornada vespertina, con condiciones y características equiparables para las variables del estudio, cada uno con número de estudiantes que oscilan entre 35 y 38 cada grupo. Se trabajó con un grupo escogido al azar para aplicar un diseño pre-experimental de grupo intacto con pre-test y pos-test, bajo los criterios de una investigación cuantitativa.

En cuanto a los instrumentos aplicados para la recolección de información se tuvo en cuenta encuestas a docentes validadas por un experto. Estas diseñadas con el objetivo de reconocer en los profesores sus concepciones teóricas y metodológicas en lo concerniente al desarrollo de competencias matemáticas y la resolución de problemas. De igual forma, se aplicó un taller diagnóstico a estudiantes, validado a nivel nacional pues su contenido se fundamenta en problemas originados en el Ministerio de Educación Nacional en el marco de la evaluación diagnóstica del Programa de Transformación de la Calidad Educativa "Todos a Aprender". El taller consta de cinco problemas que evalúan específicamente la competencia matemática de razonamiento y argumentación.

\section{Referentes Teóricos}

\section{El concepto competencia: fundamento teórico de un enfoque sobre el que se desarrolla la educación en Colombia}

El concepto competencia como tal se comenzó a estructurar en la década del sesenta con base en dos aportaciones: la lingüística de Chomsky y la psicología conductual de Skinner. Chomsky (1970) propuso el concepto de competencia lingüística como una estructura mental implícita y genéticamente determinada que se ponía en acción mediante el desempeño comunicativo (uso efectivo de la capacidad lingüística en situaciones específicas), por lo cual este autor siempre opone en el marco de su gramática generativa transformacional competencias desempeño (competence performance).

El enfoque de competencias desde lo conductual ha tenido notables desarrollos en el campo de la gestión del talento humano en las organizaciones, donde se asume con la orientación de buscar que los trabajadores posean competencias clave para que las empresas sean competitivas. Desde mediados de la década de los años noventa esta concepción de las competencias también ha sido implementada en instituciones educativas de varios países, buscando con ello formar personas con ciertas competencias que les posibiliten un mayor impacto en la inserción laboral.

\section{Algunos enfoques contextuales sobre el concepto competencia son los siguientes:}

Desde la psicolingüística, Hymes (1996) plantea el concepto de competencia comunicativa como el empleo efectivo del lenguaje y de la lingüística en situaciones específicas de comunicación, teniendo en cuenta las demandas del entorno. En una vía diferente, pero dentro del mismo enfoque, están los aportes de la psicología cultural que tienen como principal representante a Vigotsky (1985) y que han sido planteados por autores como Torrado $(1995,1998)$. Se ha propuesto que las competencias "son acciones situadas que se definen en relación con determinados instrumentos mediadores" (Hernández et al., 1998, p.14). Son acciones situadas en el sentido de que tienen en cuenta el contexto en el cual se llevan a cabo. Ahora bien, dichas acciones se dan a partir de la mente; la mente se construye en relaciones sociales y es actualizada por la cultura (Vigotsky, 1985; Brunner, 1992).

Otra línea disciplinar que ha hecho aportes significativos a las competencias es la psicología cognitiva. Es de destacar la teoría de las inteligencias múltiples de Gardner $(1987,1997)$ la cual da un apoyo teórico sustancial a la comprensión de las competencias en su dimensión cognoscitiva (Tobón, 2005). Igualmente, se tienen las contribuciones de Sternberg (1997) en torno a la inteligencia práctica, la cual se refiere a la capacidad que han de tener las personas para desenvolverse con inteligencia en las situaciones de la vida.

Todo el recorrido histórico por el concepto de competencia conduce al punto actual donde se reconoce como un concepto complejo que soporta el enfoque por competencias en el sistema educativo colombiano. De tal forma, es importante resaltar las aportaciones al respecto de Carlos Vasco (Vasco, 2003) en cuanto a los sustentos teóricos de dicho enfoque y que conllevan a que, desde el Ministerio de Educación Nacional, se defina la competencia como el conjunto de conocimientos, habilidades, actitudes, comprensiones y disposiciones cognitivas, socio-afectivas y psicomotoras apropiadamente relacionadas entre sí para facilitar el desempeño flexible, eficaz y con sentido de una actividad en 
contextos relativamente nuevos y retadores (MINISTERIO DE EDUCACIÓN NACIONAL, 2006). Las competencias son: “... un saber hacer flexible que se puede actualizar en distintos contextos... la capacidad de usar los conocimientos en situaciones distintas de aquellas en las que se aprendieron. Implica la comprensión del sentido de cada actividad, y de sus implicaciones éticas, sociales, económicas y políticas" (MINISTERIO DE EDUCACIÓN NACIONAL, 2006).

El concepto anterior, al ser el soporte del enfoque de competencias en el contexto educativo nacional, es fundamental dentro del proceso investigativo desarrollado en el presente trabajo, puesto que se vincula con otros referentes nacionales que orientan el quehacer docente como lo son los lineamientos curriculares y los estándares básicos de competencias. Además, denota la complejidad de la competencia no limitándola únicamente a lo cognitivo, sino resaltando también aspectos socio - afectivos y psicomotores, asociado a desempeño flexible y significativo en distintos contextos.

También en el marco nacional, Tobón (2006) concibe las competencias como procesos complejos de desempeño con idoneidad en un determinado contexto, con responsabilidad. Dicho concepto es pertinente, porque además de reafirmar lo expuesto por el Ministerio de Educación Nacional y sintetizarlo de manera acertada, lo complementa puntualizando dos aspectos fundamentales: idoneidad y responsabilidad. El primero relacionado con el desempeño en la resolución de problemas con criterios de eficacia, eficiencia, efectividad, pertinencia y apropiación. El segundo como el ejercicio ético de prever las consecuencias del desempeño.

Los dos últimos referentes mencionados en torno al concepto competencia son entonces los que orientaron teórica y conceptualmente el presente trabajo investigativo por su coherencia con el desarrollo educativo colombiano y su pertinencia en cuanto a la posibilidad de potenciar los aprendizajes y desempeños de los estudiantes al tener en cuenta su multidimensionalidad y los aspectos contextuales y de significatividad.

Las competencias matemáticas: aspectos y procesos que determinan el ser matemáticamente competente

A partir de reconocer las competencias como el conjunto de conocimientos, habilidades, actitudes, comprensiones y disposiciones cognitivas, socio-afectivas y psicomotoras apropiadamente relacionadas entre sí para facilitar el desempeño flexible, eficaz y con sentido de una actividad en contextos relativamente nuevos y retadores (MINISTERIO DE EDUCACIÓN NACIONAL, 2006), y sumarle a tal concepción aspectos fundamentales de idoneidad y responsabilidad, se aborda entonces lo referente a la competencia matemática y lo que implica ser matemáticamente competente. De tal forma, es pertinente tener en cuenta lo siguiente:

La competencia matemática implica la capacidad de un individuo de identificar y entender el papel que las matemáticas tienen en el mundo, para hacer juicios bien fundamentados y poder usar e involucrarse con las matemáticas. El concepto general de competencia matemática se refiere a la capacidad del alumno para razonar, analizar y comunicar operaciones matemáticas. Es, por lo tanto, un concepto que excede al mero conocimiento de la terminología y las operaciones matemáticas, e implica la capacidad de utilizar el razonamiento Matemático en la solución de problemas de la vida cotidiana (OCDE, 2009). ${ }^{1}$

La definición anterior fue relevante dentro del presente trabajo investigativo, pues reconoce como elemento fundamental en la competencia matemática el razonamiento y lo complementa con otros aspectos importantes como el análisis y la comunicación, lo que permite inferir que la argumentación se encuentra presente como elemento esencial en la competencia matemática. Además, se destaca también la relación que se establece entre tales procesos y su aplicabilidad en la resolución de problemas en diversos contextos.

Otros conceptos resaltan igualmente capacidades y habilidades asociadas a la aplicabilidad en la resolución de problemas en distintos contextos. Denótense los siguientes:

- La competencia matemática consiste en la habilidad para utilizar y relacionar los números, sus operaciones básicas, los símbolos y las formas de expresión y razonamiento matemático, tanto para producir e interpretar distintos tipos de información, como para ampliar el conocimiento sobre aspectos cuantitativos y espaciales de la realidad, y para resolver problemas relacionados con la vida cotidiana y con el mundo laboral (HEZKUNTZA, 2000).

- El conjunto de habilidades y destrezas relacionadas con el reconocimiento e interpretación de los problemas que aparecen en los diferentes ámbitos y situaciones (familiares, sociales, académicos o profesionales); su traducción al lenguaje y contextos matemáticos; su resolución empleando los procedimientos oportunos; la interpretación de los resultados y la formulación y comunicación de tales resultados (ESCAMILLA, 2008).

- Ser competente en un campo complejo como el matemático supone tener habilidad para usar los conocimientos con flexibilidad, y aplicar con propiedad lo aprendido en un contexto, a otro contexto. Se basa en un aprendizaje en el que se comprende lo aprendido. Los estudiantes deben aprender matemáticas comprendiéndolas, y construir activamente nuevos conocimientos a partir de la experiencia y de los conocimientos previos (NCTM, 2000).

Finalmente, es también importante referenciar el sustento teórico que soporta la noción de competencias matemáticas en el ámbito

OCDE. Documento PISA Colombia 2009: síntesis de resultados. ICFES, 2009. 
nacional y lo que implica ser matemáticamente competente. De tal forma es pertinente tener en cuenta que desde los Estándares básicos de competencias para el área de matemática, se concibe el ser matemáticamente competente como:

- Formular, plantear, transformary resolver problemas a partir de situaciones de la vida cotidiana, de las otras ciencias y de las matemáticas mismas. Ello requiere analizar la situación; identificar lo relevante en ella; establecer relaciones entre sus componentes y con situaciones semejantes; formarse modelos mentales de ella y representarlos externamente en distintos registros; formular distintos problemas, posibles preguntas y posibles respuestas que surjan a partir de ella. Este proceso general requiere del uso flexible de conceptos, procedimientos y diversos lenguajes para expresar las ideas matemáticas pertinentes y para formular, reformular, tratar y resolver los problemas asociados a dicha situación. Estas actividades también integran el razonamiento, en tanto exigen formular argumentos que justifiquen los análisis y procedimientos realizados y la validez de las soluciones propuestas.

- Utilizar diferentes registros de representación o sistemas de notación simbólica para crear, expresar y representar ideas matemáticas; para utilizar y transformar dichas representaciones y, con ellas, formular y sustentar puntos de vista. Es decir dominar con fluidez distintos recursos y registros del lenguaje cotidiano y de los distintos lenguajes matemáticos.

- Usar la argumentación, la prueba y la refutación, el ejemplo y el contra ejemplo, como medios de validar y rechazar conjeturas, y avanzar en el camino hacia la demostración.

- Dominar procedimientos y algoritmos matemáticos y conocer cómo, cuándo y por qué usarlos de manera flexible y eficaz.

Todo esto conlleva a reconocer la competencia matemática como un conjunto de conocimientos y habilidades matemáticas, actitudes, comprensiones y disposiciones cognitivas, socio-afectivas y psicomotoras apropiadamente relacionadas entre sí para facilitar desempeño desde el razonamiento, la argumentación, la comunicación, representación, modelación y el planteamiento y resolución de problemas en diversos contextos.

\section{Competencias matemáticas y resolución de problemas}

Como ya se mencionó, el ser matemáticamente competente se relaciona con el dominio de diversos procesos matemáticos relacionados entre sí y que confluyen en la actividad matemática y en el desempeño del estudiante en diversos contextos. Sin embargo, es relevante puntualizar en el aspecto siguiente:

Ser matemáticamente competente, entre otros aspectos, supone... formular, plantear, transformar y resolver problemas a partir de situaciones de la vida cotidiana, de las otras ciencias y de las matemáticas mismas. Ello requiere analizar la situación; identificar lo relevante en ella; establecer relaciones entre sus componentes y con situaciones semejantes; formarse modelos mentales de ella y representarlos externamente en distintos registros; formular distintos problemas, posibles preguntas y posibles respuestas que surjan a partir de ella. Este proceso general requiere del uso flexible de conceptos, procedimientos y diversos lenguajes para expresar las ideas matemáticas pertinentes y para formular, reformular, tratar y resolver los problemas asociados a dicha situación. Estas actividades también integran el razonamiento, en tanto exigen formular argumentos que justifiquen los análisis y procedimientos realizados y la validez de las soluciones propuestas (Ministerio De Educación Nacional, 2006).

Tal planteamiento fue fundamental dentro del presente trabajo, pues apropia cabalmente el concepto de competencia y a su vez incluye otros aspectos complementarios como la modelación, representación, razonamiento y argumentación; siendo estos últimos ejes centrales dentro del objeto de estudio al que refiere esta investigación.

Ahora bien, es también importante referirse a la resolución de problema desde los procesos generales de la actividad matemática, lo que implica una mirada específica que lo denota como un subproceso que permite identificar capacidades, habilidades, actitudes, entre otros, que potencian los desempeños desde las competencias.

La resolución de problemas es un proceso presente a lo largo de todas las actividades curriculares de matemáticas y no una actividad aislada y esporádica; más aún, podría convertirse en el principal eje organizador del currículo de matemáticas, porque las situaciones problema proporcionan el contexto inmediato en donde el quehacer matemático cobra sentido, en la medida en que las situaciones que se aborden estén ligadas a experiencias cotidianas $y$, por ende, sean más significativas para los alumnos. Estos problemas pueden surgir del mundo cotidiano cercano o lejano, pero también de otras ciencias y de las mismas matemáticas, convirtiéndose en ricas redes de interconexión e interdisciplinariedad (Ministerio De Educación Nacional, 2006).

Se denota a partir de lo anteriormente expuesto que la resolución de problemas puede ser entendida de manera transversal en los procesos educativos y además posibilita la significatividad del aprendizaje al aprovechar diferentes contextos.

La formulación, el tratamiento y la resolución de los problemas suscitados por una situación problema permiten desarrollar una actitud mental perseverante e inquisitiva, desplegar una serie de estrategias para resolverlos, encontrar resultados, verificar e interpretar lo razonable de ellos, modificar condiciones y originar otros problemas. Es importante abordar problemas abiertos donde sea posible encontrar múltiples soluciones o tal vez ninguna. También es muy productivo experimentar con problemas a los cuales les sobre o les falte información, o con enunciados narrativos o incompletos, para los que los estudiantes mismos tengan que formular las preguntas. Más bien que la resolución de multitud de problemas tomados de los textos 
escolares, que suelen ser sólo ejercicios de rutina, el estudio y análisis de situaciones problema suficientemente complejas y atractivas, en las que los estudiantes mismosinventen, formulen y resuelvan problemas matemáticos, es clave para el desarrollo del pensamiento matemático en sus diversas formas (Ministerio De Educación Nacional, 2006).

Se resalta la importancia de la resolución de problemas en lo referente al desarrollo del pensamiento matemático en sus diversas formas, desde el tratamiento de situaciones problémicas que trasciendan la ejercitación e impliquen el análisis, el razonamiento, la modelación, la argumentación, entre otras habilidades de pensamiento, que potencien de forma significativa los aprendizajes y desarrollen de tal forma las competencias en los estudiantes.

Después de haber mirado la resolución de problemas como proceso, es pertinente hacer una revisión conceptual que oriente la forma de abordar el objeto de estudio y coadyuve a delimitar el campo de la investigación.

Problema, según Parra (1990) establece que "un problema lo es en la medida en que el sujeto al que se le plantea (o que se plantea él mismo) dispone de los elementos para comprender la situación que el problema describe y no dispone de un sistema de respuestas totalmente constituido que le permita responder de manera inmediata".

Dentro de la Psicología cognitiva se puede tomar como punto de partida la definición de problema aportada por H.A. Simón (1978): "una persona se enfrenta a un problema cuando acepta una tarea, pero no sabe de antemano como realizarla. Aceptar una tarea implica poseer algún criterio que pueda aplicarse para determinar cuándo se ha terminado la tarea con éxito" o también la que proponen Chi y Glaser (1986): "un problema es una situación en la que se intenta alcanzar un objetivo y se hace necesario un medio para conseguirlo".

De acuerdo a las anteriores definiciones un problema va acompañado siempre de una cierta incertidumbre y en ese sentido es posible llamar "resolución de problemas" al proceso mediante el cual la situación incierta es clarificada implicando siempre la aplicación de conocimientos por parte del sujeto que resuelve.

Desde una perspectiva histórico-psicológica ha habido dos aportes respecto al origen de la resolución de problemas: la del paradigma asociacionista (el problema se resuelve por ensayo / error, después de haber resuelto una serie de problemas similares, sin embargo este tratamiento es superficial y confuso) y la segunda la Psicología de la Gestalt (va más allá de una mecánica reproductiva, la resolución implica relacionar diferentes aspectos y captar como todas las partes del problema encajan para satisfacer las exigencias del objetivo, lo que implica reorganizar los elementos de la situación problemática y en consecuencia resolver el problema (R. Mayer,1986).
La corriente más fuerte y con mayor influencia en el campo de la resolución de problemas, dentro del marco de la Psicología cognitiva (enmarcada en la corriente denominada constructivismo), es la conocida con el nombre de Procesamiento de la información desarrollada desde hace unos 20 años a partir de las aportaciones de A.Newell y H.A. Simón.

Para el trabajo investigativo presente se tomaron como referentes teóricos las aportaciones de G. Polya, G. (1969) quien considera que las principales fases para la resolución de problemas son las siguientes:

- Comprender el problema.

- Captar las relaciones que existen entre los diversos elementos con el fin de encontrar la idea de la solución y poder trazar un plan.

- Poner en ejecución el plan.

- Volver atrás una vez encontrada la solución, revisarla y discutirla.

El propone tres preguntas generales que el profesor debe formular a los estudiantes, para ayudarlo a resolver un problema en particular y a desarrollar habilidades en la solución de problemas en general. Estas preguntas son: ¿Cuál es la incógnita?, ¿Cuáles son los datos?, ¿Cuál es la condición?

Polya, G. (1969) en su libro ¿Cómo plantear y resolver problemas? identifica los términos ejercicios y problemas y revela la existencia de 4 tipos de problemas:

- Problemas por resolver, cuyo propósito es descubrir cierto objeto, la incógnita del problema.

- Problemas por demostrar, aquí el propósito es "mostrar, de un modo concluyente, la exactitud o falsedad de una afirmación claramente enunciada".

- Problemas de rutina, es todo aquel problema que se puede resolver ya sea sustituyendo simplemente nuevos datos en el lugar de los de un problema ya resuelto, ya sea siguiendo paso a paso, sin ninguna originalidad, la traza de algún viejo ejemplo.

- Problemas prácticos o de aplicación a la práctica.

Luego un ejercicio es un problema si y sólo si la vía de solución es desconocida para la persona; reconociendo en el proceso de enseñanza la posibilidad de las personas de resolver problemas matemáticos como una capacidad específica que se desarrolla en el individuo en dicho proceso. Una situación problémica puede ser construida como un ejercicio o un problema matemático en dependencia de los datos que se ofrecen en el mismo los cuales estarán en correspondencia con los conocimientos que el estudiante debe utilizar en su solución. Entonces, solucionar un problema es darle cumplimiento al objetivo de todas las acciones que lo 
caracteriza como un ejercicio en la enseñanza de la matemática. En el proceso de resolver el problema se debe ejecutar cuatro acciones: comprender el problema, analizar el problema, solucionar el problema y evaluar la solución del problema.

\section{Competencias matemáticas: razonamiento y argumentación}

Desde los lineamientos curriculares y los estándares básicos de competencias para el área de matemáticas se enuncian una serie de procesos generales que explicitan el ser matemáticamente competente, estos son: formular y resolver problemas; modelar procesos y fenómenos de la realidad; comunicar; razonar, y formular comparar y ejercitar procedimientos y algoritmos.

Para este trabajo investigativo se analizo particularmente el proceso de razonamiento puesto que corresponde a la competencia que se pretende potenciar, aunque es pertinente aclarar que los procesos matemáticos en términos de competencias deben verse como un todo integrado que coadyuve a la resolución de problemas en diversos contextos.

El desarrollo del razonamiento lógico empieza en los primeros grados apoyado en los contextos y materiales físicos que permiten percibir regularidades y relaciones; hacer predicciones $y$ conjeturas; justificar o refutar esas conjeturas; dar explicaciones coherentes; proponer interpretaciones y respuestas posibles y adoptarlas o rechazarlas con argumentos y razones. Los modelos y materiales físicos y manipulativos ayudan a comprender que las matemáticas no son simplemente una memorización de reglas y algoritmos, sino que tienen sentido, son lógicas, potencian la capacidad de pensar y son divertidas. En los grados superiores, el razonamiento se va independizando de estos modelos y materiales, y puede trabajar directamente con proposiciones y teorías, cadenas argumentativas e intentos de validar o invalidar conclusiones, pero suele apoyarse también intermitentemente en comprobaciones e interpretaciones en esos modelos, materiales, dibujos y otros artefactos (MINISTERIO DE EDUCACION NACIONAL, 2006).

El reconocer cómo el razonamiento matemático se desarrolla permite visionar estrategias pedagógicas que contribuyan a su potenciamiento, privilegiando escenarios significativos que generen en los estudiantes motivación y faciliten los procesos de aprendizaje.

Es conveniente que las situaciones de aprendizaje propicien el razonamiento en los aspectos espaciales, métricos y geométricos, el razonamiento numérico y, en particular, el razonamiento proporcional apoyado en el uso de gráficas. En esas situaciones pueden aprovecharse diversas ocasiones de reconocer y aplicar tanto el razonamiento lógico inductivo y abductivo, al formular hipótesis o conjeturas, como el deductivo, al intentar comprobar la coherencia de una proposición con otras aceptadas previamente como teoremas, axiomas, postulados o principios, o intentar refutarla por su contradicción con otras o por la construcción de contraejemplos (MINISTERIO DE EDUCACION NACIO$N A L, 2006)$.

Se denota entonces la importancia de las situaciones de aprendizajes, las cuales deben propender por lo significativo en aras de incentivar el razonamiento lógico, tanto inductivo como deductivo, en el desarrollo de los distintos tipos de pensamiento.

Ahora bien, la argumentación, aunque puede parecer a priori una competencia más asociada al lenguaje, es igualmente relevante en lo que concierne a los procesos matemáticos. Por tanto, el ICFES agrupa las competencias y expone los grupos siguientes:

- Razonamiento y argumentación

- Comunicación, representación y modelación

- Planteamiento y resolución de problemas

Como se mencionó ya, desde la investigación presente se abordo la competencia de razonamiento y argumentación, por tanto, finalmente, acorde al ICFES, se define así:

La competencia de razonamiento y argumentación están relacionadas, entre otros, con aspectos como el dar cuenta del cómo y del porqué de los caminos que se siguen para llegar a conclusiones, justificar estrategias y procedimientos puestos en acción en el tratamiento de situaciones problema, formular hipótesis, hacer conjeturas, explorar ejemplos y contraejemplos, probar y estructurar argumentos, generalizar propiedades $y$ relaciones, identificar patrones y expresarlos matemáticamente y plantear preguntas, reconocer distintos tipos de razonamiento $y$ distinguir y evaluar cadenas de argumentos (ICFES, 2009).

En síntesis, las competencias, aunque no pueden ser entendidas desligadas unas de otras en un contexto práctico, en el ejercicio pedagógico puede mirarse de forma puntual en aras de favorecer su desarrollo desde sus características específicas, propiciando metodologías que convengan su potenciamiento.

\section{Referencias}

Ausubel, D. P.; Novak, J. D. y Hanesian, H. (1983). Psicología educativa: Un punto de vista cognoscitivo (2a. ed.). México: Trillas.

Bustamante, G. (2003). El concepto de competencia III. Un caso de recontextualización: Las "competencias" en la educación colombiana. Bogotá: Sociedad Colombiana de Pedagogía.

Chomsky, N. (1970). Aspectos de la teoría de la sintaxis. Madrid: Editorial Aguilar.

Gardner, H. (1987). Las estructuras de la mente. La teoría de las inteligencias múltiples. México: Fondo de Cultura Económica.

Hymes, D. (1996). Acerca de la competencia comunicativa. Forma y Función, 9.Departamento de Lingüística. Bogotá: Universidad Nacional.

ICFES (2009). Informe Icfes: Colombia en PISA 2009. Síntesis de resultados. Bogotá: ICFES. 
ICFES. (2009). Lineamientos generales SABER 2009, grados 5 y 9. Bogotá: ICFES.

López, A. S., \& Trigo, L. M. S. (2006). Desarrollo de Episodios de Comprensión Matemática: Estudiantes de bachillerato en procesos de resolución de problemas. Revista Mexicana InvestigaciónEducativa,11(31),1389-1422. Retrievedfrom http:// search.proquest.com/docview/199269012?accountid=44025

López Silva, Luz Stella. (2011). La clase para pensar. Colombia: Universidad del Norte.

Ministerio de Educación Nacional. (2006). Estándares básicos de Competencia en matemáticas. Bogotá: Ministerio de Educación Nacional.

Ministerio de Educación Nacional (1998). Matemáticas. Lineamientos curriculares. Bogotá.

Novak, J. D. y Gowin, B. (1988). Aprendiendo a aprender. Barcelona: Martínez Roca

OCDE (en prensa). Tomorrow's skills today. Student performance in PISA 2009. OCDE.

Polya G. (1981). Cómo plantear y resolver problemas. México: Trillas.

Quiroga, B. G., Coronado, A., \& Quintana, L. M. (2011). Formación y desarrollo de competencias matemáticas: Una perspectiva teórica en la didáctica de las matemáticas*/Formation and development of mathematicalcompetences: A theoreticalperspective in thedidactics of mathematics/Formation et développement de compétencesmathématiques: Une perspectivethéoriquedans la didactique des mathématiques. Revista Educación y Pedagogía, 23(59),159-175.Retrievedfrom http://search. proquest.com/docview/1240994387?accountid $=44025$

Sternberg, R.J. (1997). Successful intelligence. New York: Simon and Shuster.

Tobón, S. (2004). Formación basada en competencias: Pensamiento complejo, diseño curricular y didáctica. Bogotá: ECOE.

Tobón, S. (2006). Las competencias en la educación superior. Políticas de calidad. Bogotá: ECOE.

Vasco, C. E. (1998). "Visión de conjunto de la pedagogía de las matemáticas como disciplina en formación". En: Revista Matemática-Enseñanza Universitaria. Vol. 7. No. 1, 75-88.

Vasco, C. E. (1994). Un nuevo enfoque para la didáctica de las matemáticas (2a. ed., 2 vols.). Bogotá: Ministerio de Educación Nacional.

Vigotsky, L. (1985). Pensamiento y lenguaje. Buenos Aires: La Pléyade.

Wiske, M. S. (Comp.). (2003). La enseñanza para la comprensión. Vinculación entre la investigación y la práctica. Buenos Aires, Barcelona, México: Paidós 